\title{
Inheritance of paper-cut art in the design of clothing fabric
}

\author{
Yancong Miao \\ Hebei University of Science and Technology, College of Textile and \\ apparel,Shijiazhuang,050018,China \\ 275929180@qq.com
}

Keywords:Clothing fabrics; Design; Paper-cut; Inheritance

Abstract. As Chinese paper-cut art of local culture, implies a deep cultural heritage, has extensive flat constituent elements and adornment sex is extremely strong. Through the paper-cut art many aspects cultural reference, apply paper-cut art in the design of the clothing fabric, and nationality color in the collocation of format, stylized creation approach two aspects of the formation of Innovation clothing fabric design philosophy, to promote China's clothing fabric design nationality process.

\section{Introduction}

The fabric design of clothing is an important factor of costume design, not only conveying the era of clothing main body in the consciousness, but also is the supporting body of cultural connotation. The traditional clothing fabric design concerned only functional and technical, ignoring its cultural phase matching. With the rapid development of science and technology, and inheritance to the concept of traditional art and thinking, under the new situation of clothing fabric development should reflect the culture characteristics of consumer groups, for the fabric design of clothing provides a wider space. Paper-cut art form elements and cultural connotations, make up for the original of the fabric design of clothing lack of cultural background. From the color of nationality in collocation,stylized creation approach way two aspects of creative paper-cut art can be incorporated into clothing fabric design .

\section{Paper-cut art is a multidimensional culture bearer}

\section{The theory of Yin and Yang in paper-cut art}

The paper-cut modeling essence nature is the positive and negative, which is commonly referred to as the "yin and yang" and "yin and yang theory" is China's unique cultural phenomenon, reveal with the generation and change in the world is the interaction between Yin and Yang. Yin and Yang theory is precisely to improve the design of the transmission of cultural information through ideological and practical functions of cultural message function.

\section{The appeal of auspicious culture in paper-cut art}

Chinese traditional philosophy pays attention to the unity of nature and a satisfactory thought, its composition form and content are happy happy reunion creation intention. Paper-cut art is a representative of auspicious culture, the homonym techniques specific symbol semantics reflects the peace auspicious and symbolic meaning, more embodies the auspicious complete design concept.

\section{The decorative unique aesthetic function in paper-cut art}

Chinese paper-cut art is due to the geographical distribution of different, presents its aesthetic style has different features. South area of paper-cut art style because of its shape ethereal clear 
structure, exquisite and delicate and refined, the northern region of paper-cut art often seen large area color contrast, modeling structural area are strong, give a person bold simple style. The aesthetic function of the art of paper cutting has become the main performance of modern society.

\section{The unique modeling of art form in paper-cut art}

Paper-cut art exquisite on operating means, such as a rectangle like blue bricks, a circular like complete the moon, line as fine as whiskers, serrated as incomplete, the tip as the awn of wheat. The mood in accordance with the expression of creative design thinking way and pay attention to the mood and scene combination, is beyond the reality ideal creation method. Traditional paper-cut art carry on ancient Chinese design thinking, main show is paring down. Unique composition planar form is the theme of paper-cut art, which will be the objective thing or the illusion of the three-dimensional state to do parallel processing, expanded into flat art form.

\section{Paper-cut art characteristics into the Clothing fabric design}

\section{Nationality color in the collocation of format}

The colors of the paper-cut art with reference to the Chinese traditional mode of the "five colors", according to the traditional concept of the five parties, formed white tiger in west, cyan dragon in East, Xuanwu in South, vermilion bird in north, the middle is for the emperor yellow. Common traditional paper-cut, mostly red and black, through the evolution of history in modern times. Color paper-cut art often appear in the Yuxian Hebei, Guangdong Foshan, Shanbei and other places. Such as paper cutting master ShuLan $\mathrm{Ku}$ is a typical representative of the Chinese color paper-cut. As figure 1 and figure 2 shows.

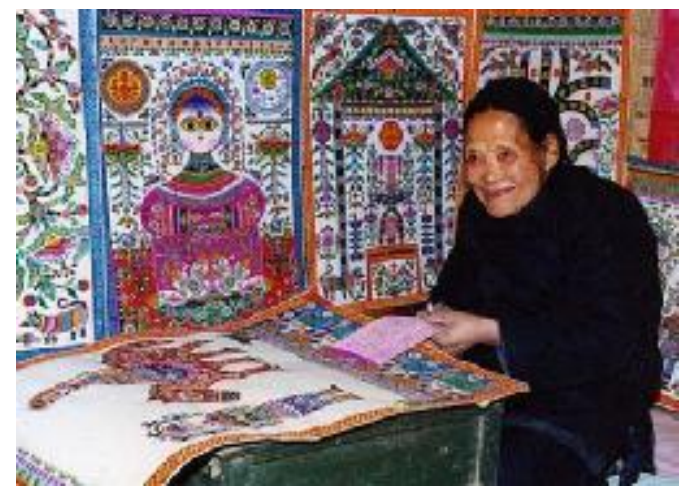

Figure 1. paper cutting master ShuLan Ku lady"by KuShuLan

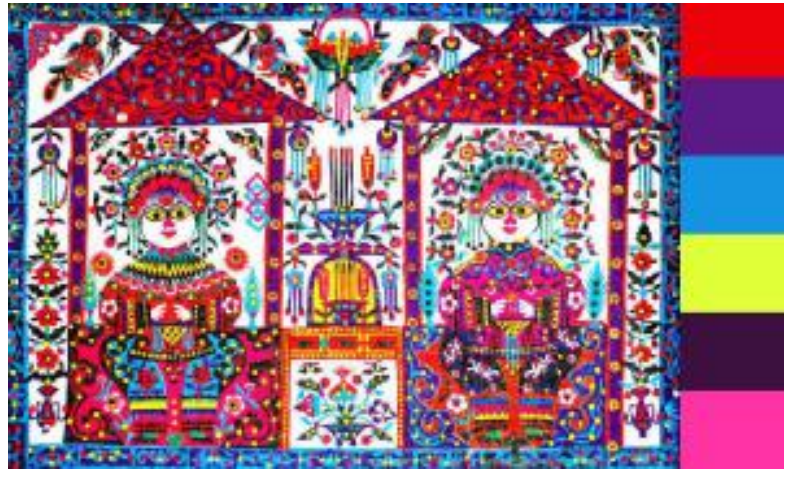

Figure 2. colorful paper-cutting works "cut flower

The paper-cut art monochromatic color format is only a kind of pure color format, common colors are red, yellow, blue, green and so on. This color format color, color sense is strong, give a person with simple but elegant and elegant visual experience. Using this monochromatic color scheme in clothing fabric design need to be in shape and pattern design is exquisite and accurate features in the design, reasonable use of dot, line, area to profoundly shape the image, to create a concise and elegant the overall effect and strong decorative details.

The color scheme with color Paper-cut art is also known as the complex color format, is the picture in the use of two or more than two color matching format. Traditional paper-cut art often uses the color is the traditional "five elements" form, i.e., black, white, red, yellow and green. This complex color matching format using "Image" performance method, relying only on the objective image of the subconscious reflect subjective initiative, which is not attached to the existing color theory, just follow their psychological perception and the traditional "yin and yang" color Ideas in 
Chinese. In the garment fabric design, the use of this complex color scheme for color design, the color with intense collocation, make the picture with strong ethnic characteristics. The complexity of this color collocation model is shown in figure 2 .

\section{Stylized creation approach}

Stylized creation was between the constituent elements to establish a stable styling pattern and internal relations between elements, stylized art of composition is mainly reflected in the "symmetrical cut", "folded cut", "random styling method", "destroy the original the shape of method", "the method of full shape", which are typical features stylized. This stylized picture composition using the "repeat" this visual forms, to enhance the effect of visual decoration. Paper-cut stylized styling mainly through symmetry and balance, and repeated and coherent, rhythm and rhythm and other forms of expression, embodied in the form to constituent elements showing aesthetic feeling. Common paper-cut art stylized modeling way: intaglio modeling, Yang carved modeling, Yin and Yang modeling, the silhouette modeling .

Intaglio modeling is originated from the ancient yin-yang theory, commonly used intaglio to hollow out paper-cut modeling, the characteristics of this way of modeling is to get rid of the outline of the graph, leave around the contour surface block structure to shape the image. This kind of negative space shape, structure block surface effect is strong, the overall effect have strong a sense of weight. In the clothing fabric design this kind of the intaglio modeling is suitable for the dignified design theme. As figure 3 shows.

Yang carved modeling is paper-cut bulge part, the effect produced is bulge shape modeling features, which is characterized by retained contour line, the contour line around block surface away, and with the "lines and lines connected, area and area disconnect" visual effects. Carved Yang Styling the overall effect is more transparent, so that the screen point, line and plane smooth performance and density equalization, contrast to the line sense strong. In garment fabrics design for a more relaxed and lively design theme, its focus on pay attention to exquisite and depicting fine downy picture effect, see more at lace fabric design. As figure 4 shows.

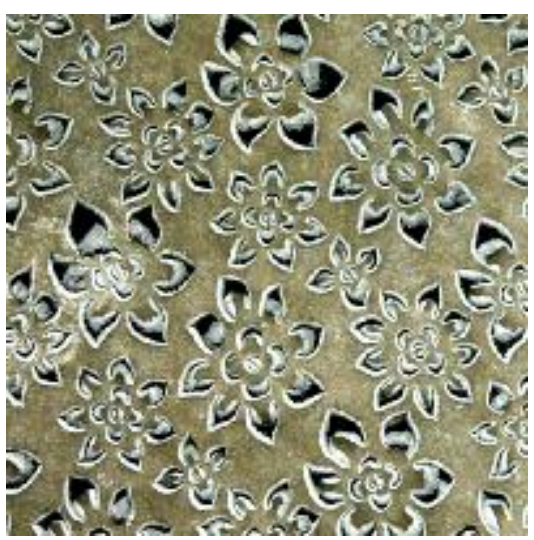

Figure 3. Intaglio modeling of garment fabrics design design

Modeling approach of Yin and Yang is the most important form of paper cutting art, China is important manifestation of the traditional doctrine of Yin and Yang. This creation approach combined with the combination of the imaginary and the real, according to Chinese the traditional design thinking, "nature and humanity","Yin and Yang coordination" simple creation ideas, illusions and realities in order to create a graceful image of complement each other.

Silhouette modeling is a kind of special form of expression of paper-cut art, its extremely concise form of art expression is only depicted outline, not deliberately portrayed is one of the most concise form of art. 
Silhouette modeling use in garment fabrics design to simplify picture structure, and generates multiple the association of ideas, but also the pursuit of a successful and new ideas design.

\section{Conclusion}

The Chinese paper-cut art contains profound national culture accumulation, as a Chinese national symbolic visual performance is important source of Chinese modern art design field. In clothing fabric design needs to multidimensional grasp the form traditional characteristics paper-cut art,in the designers express true feelings at the same time, we must good at continuous researching paper-cut of cultural connotation and creation methods in the clothing fabric design.

\section{References}

[1] Shuhua Bie, The form of paper cutting art and symbolic meaning of the Shandong Normal University,2010,pp.7-24.

[2] Xianru $\mathrm{Xu}$, Jiyao Ling, Changes of the functional meaning of Chinese folk paper-cut art and culture, Journal of Northwest University For Nationalities (PHILOSOPHY AND SOCIAL SCIENCE EDITION) .6 (2009)78-83.

[3] Hue Liang, Jiaojiao Liu, Symbolic patterns of paper-cut, Hundred Schools in Arts, 7 (2011)165-168.

[4] Jiaojiao Liu, The style of paper-cut art of design in modern woman's innovative use of, Jiangnan University Master's thesis. 2011, pp. 35-43.

[5]He Qi, Yin and Yang of China culture, Journal of literature and history expo theory,4(2009)22-25.

[6] Hanxia Bai, Theory of characteristics and genetic analysis of China's folk paper-cut art style, Master thesis, central academy of fine arts. 2003,pp.16-28. 